Article

\title{
Forge-Hardened TiZr Null-Matrix Alloy for Neutron Scattering under Extreme Conditions
}

\section{Takuo Okuchi $^{1, *}$, Akinori Hoshikawa ${ }^{2}$ and Toru Ishigaki ${ }^{2}$}

1 Institute for Study of the Earth's Interior, Okayama University, 827 Yamada, Misasa, Tottori 682-0193, Japan

2 Frontier Research Center for Applied Atomic Sciences, Ibaraki University, 162-1 Shirakata, Tokai, Naka, Ibaraki 319-1106, Japan; E-Mails: akinori.hoshikawa.eml@ vc.ibaraki.ac.jp (A.H.); toru.ishigaki.01@vc.ibaraki.ac.jp (T.I.)

* Author to whom correspondence should be addressed; E-Mail: okuchi@ misasa.okayama-u.ac.jp; Tel.: +81-858-43-1215; Fax: +81-858-43-2184.

Academic Editor: Klaus-Dieter Liss

Received: 8 October 2015 / Accepted: 7 December 2015 / Published: 9 December 2015

\begin{abstract}
For neutron scattering research that is performed under extreme conditions, such as high static pressures, high-strength metals that are transparent to the neutron beam are required. The diffraction of the neutron beam by the metal, which follows Bragg's law, can be completely removed by alloying two metallic elements that have coherent scattering lengths with opposite signs. An alloy of $\mathrm{Ti}$ and $\mathrm{Zr}$, which is known as a TiZr null-matrix alloy, is an ideal combination for such purposes. In this study, we increased the hardness of a TiZr null-matrix alloy via extensive mechanical deformation at high temperatures. We successfully used the resulting product in a high-pressure cell designed for high-static-pressure neutron scattering. This hardened TiZr null-matrix alloy may play a complementary role to normal TiZr alloy in future neutron scattering research under extreme conditions.
\end{abstract}

Keywords: neutron scattering; null-matrix alloy; high pressure; TiZr 


\section{Introduction}

To conduct neutron scattering research under extreme conditions, such as neutron diffraction at high static pressures, metals that are transparent to neutrons and have a large mechanical strength are required for creating suitable sample environments [1]. The ideal metals for such applications should have small coherent and incoherent scattering lengths to reduce both the Bragg diffraction and background signals. The coherent scattering length of a metal can be minimized by preparing an alloy of two elements that have coherent scattering lengths with opposite signs. Such alloys are collectively called "null-matrix alloys", and the representatives are $\mathrm{Mn}(68$ atom \%) and $\mathrm{Cu}$ (32 atom \%), which are ductile and soft [2], and $\mathrm{Ti}$ (68 atom \%) and $\mathrm{Zr}$ (32 atom \%), which are brittle and hard [3]. The latter $\mathrm{Ti}_{68} \mathrm{Zr}_{32}$ alloy (hereinafter referred to as $\mathrm{TiZr}$ ) has been extensively used to fabricate metal gaskets for compressing samples in high-pressure environments, which are essential components of typical high-pressure cells designed for crystal structure analysis [4-6]. TiZr has the highest mechanical strength as a null-matrix alloy, which is roughly comparable to that of stainless steels [1]. TiZr also exhibits a moderate elongation of $6 \%-8 \%$ to be used in the aforementioned application [7].

In this study, by considering the unique properties of TiZr, we attempted to further enhance its mechanical properties while maintaining its null-scattering behavior via extensive mechanical deformation at high temperatures at ambient pressure. After preparing and analyzing the resulting product, we tested the applicability of the treated alloy to high-static-pressure experiments by using it in a high-pressure cell designed for neutron scattering.

\section{Experimental Methods}

While a structural phase transition of TiZr null-matrix alloy was observed to occur as the effect of high static pressure [8], the effects of extensive deformation on its physical properties has not yet been studied. The metallographic textures and mechanical properties of $\mathrm{Ti}-\mathrm{Zr}$ two-component $\alpha$-alloy system can be modified and improved by subjecting them to plastic deformations (via mechanical forging) [9]. Therefore, here we prepared a cast billet of TiZr and applied extensive plastic deformation for it at ambient pressure [10,11]. A total of $85 \mathrm{~kg}$ of the TiZr alloy was cast and forged as a custom-made commercial order to Daido Bunseki Research, Inc. (Nagoya, Japan). In detail, the weighted Ti- and Zr-containing precursors were first melted together in a plasma progressive-casting furnace, where an electrode for the subsequent vacuum arc remelting (VAR) procedure was prepared. The electrode was then subjected to the VAR procedure, which produced a homogenized ingot after the remelting and recooling. The appearance of the ingot is shown in Figure 1, while the composition is shown in Table 1. 


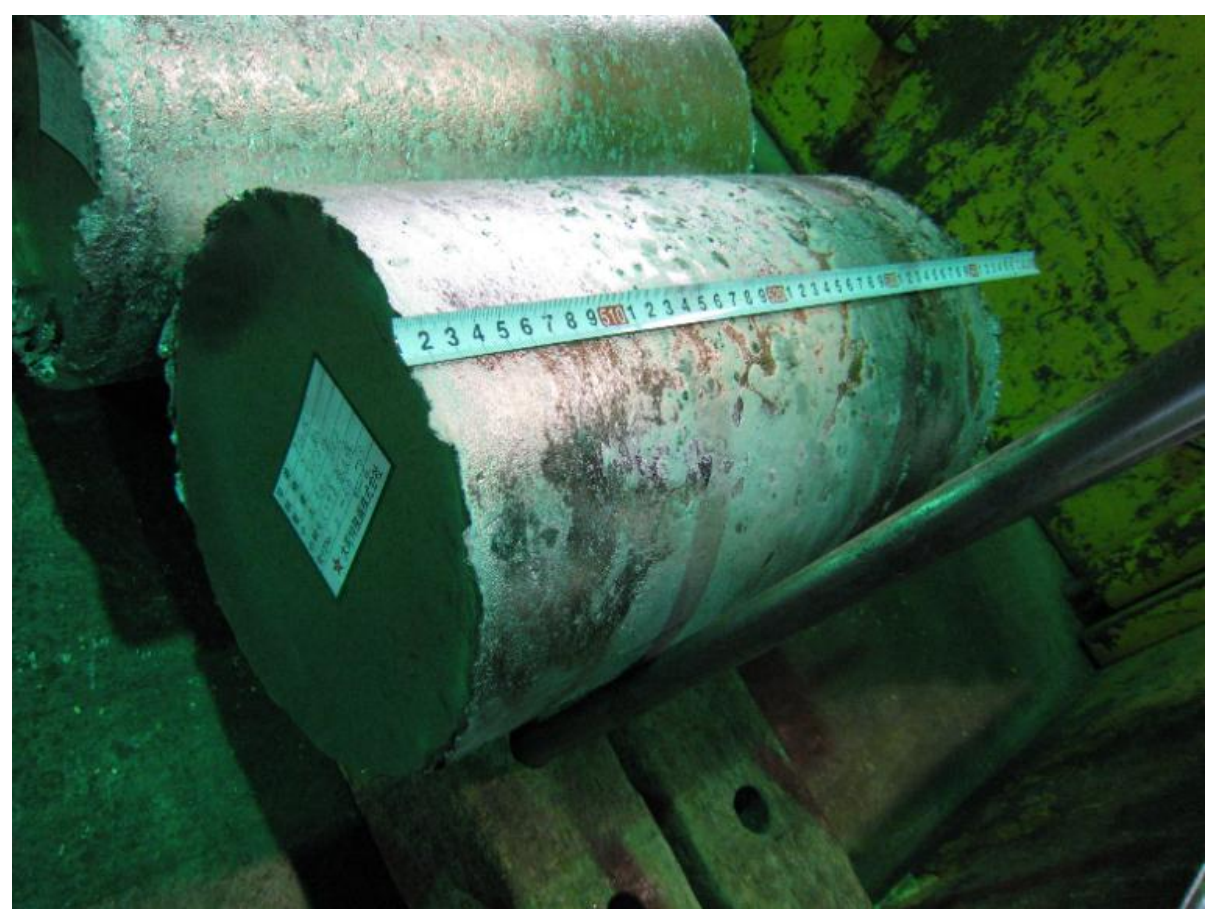

Figure 1. Photograph of the VAR-fabricated TiZr ingot, which was $240 \mathrm{~mm}$ in diameter, $384 \mathrm{~mm}$ in length, and $85.4 \mathrm{~kg}$ in weight.

Table 1. Chemical composition of the vacuum arc remelting (VAR)-fabricated TiZr ingot.

\begin{tabular}{cccccccc}
\hline \multicolumn{7}{c}{ Composition wt. \% } \\
\hline $\mathrm{Zr}$ & $\mathrm{C}$ & $\mathrm{Al}$ & $\mathrm{B}$ & $\mathrm{O}$ & $\mathrm{N}$ & $\mathrm{Fe}$ & $\mathrm{Ti}$ * \\
47.1 & 0.008 & 0.01 & $<0.01$ & 0.053 & 0.004 & 0.03 & 52.8 \\
\hline \multicolumn{10}{c}{ * Ti as the balance. }
\end{tabular}

The VAR-fabricated TiZr ingot was then polished to remove any macroscopic cracks in the surface. An evacuated steel sheath was used to subject the billet to vacuum conditions during the forging process, which is an effective method for preventing the oxidation of the ingot. The ingot-containing steel sheath was heated to approximately $1100{ }^{\circ} \mathrm{C}$ and then forged immediately. The diameter of the ingot was then reduced to approximately $100 \mathrm{~mm}$ as the temperature decreased to around $700{ }^{\circ} \mathrm{C}$.

The thinned ingot (Figure 2) was then cut, re-sealed in another steel sheath, and forged again into a number of cylinders with diameters of 20-30 $\mathrm{mm}$. This process was conducted at temperatures within the $\beta$-phase field, and the final products were air-cooled into the $\alpha$-phase [12]. The final products had a forging ratio $\left(F_{0} / F\right)$ of more than 60 , or $e=\ln \left(F_{0} / F\right)=4-5$, where $F_{0}$ and $F$ are the initial and final cross-sectional areas, respectively. These values were sufficient to produce the desired metallographic texture. Hereinafter, we will refer to the final products as "f-TiZr", where the "f" indicates that the resulting products were both "forged" and "finely textured". 


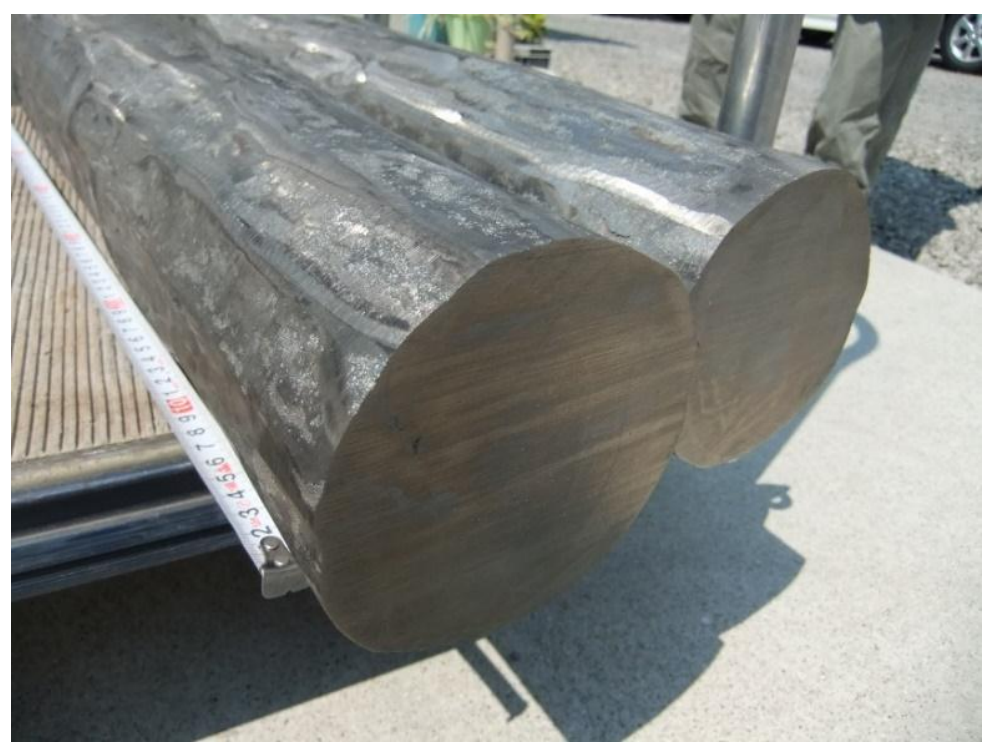

Figure 2. Photograph of the forged ingot that is approximately $100 \mathrm{~mm}$ in diameter, which was mechanically thinned from the VAR-fabricated TiZr ingot that was $240 \mathrm{~mm}$ in diameter.

The metallographic textures and elemental concentration maps of the f-TiZr alloy were obtained with a field-emission scanning electron microscopy (FE-SEM; JEOL JSM-7001F, Akishima, Tokyo, Japan) setup that was capable of performing energy dispersive X-ray spectroscopy (EDS). The micro-Vickers hardness of the alloy was measured using a micro hardness tester with $300 \mathrm{~g}$ to $500 \mathrm{~g}$ of the applied force (Mitsutoyo HM-221, Kawasaki, Japan). Powder X-ray diffraction (XRD; Rigaku Smartlab, Akishima, Tokyo, Japan) was used to analyze the crystal structure, while neutron powder diffraction (NPD) was used to evaluate the coherent neutron scattering. The XRD patterns were obtained by using the para-focusing method with $\mathrm{Cu} \mathrm{K} \alpha_{1}$ radiation operated at $40 \mathrm{kV}$ and $30 \mathrm{~mA}$. The NPD patterns were obtained with an iMATERIA pulsed-neutron powder diffractometer at the Materials and Life Science Experimental Facility of the Japan Proton Accelerator Research Complex (J-PARC; Tokai, Japan). The neutron diffractometer covered a wide range of $d$-values $(0.26-40 \AA)$ in the double-frame operating mode [13].

We expected that the toughness and elongation of the f-TiZr would not be sufficient for certain applications that involve extensive deformations of the metal. Therefore, some of the samples were cut from the forged TiZr ingot with $100 \mathrm{~mm}$ in diameter (Figure 2) and were annealed and furnace-cooled at $660{ }^{\circ} \mathrm{C}$ for $6 \mathrm{~h}$ in an Ar atmosphere using a gas convertible vacuum furnace (FUA112DB, Advantec Toyo Kaisya, Ltd., Tokyo, Japan), which is an effective method for increasing the toughness and elongation of the alloy while reducing its hardness [9].

\section{Results and Discussion}

Figure 3 shows typical cross-sectional SEM images of the VAR-fabricated TiZr ingot before it was forged. The back-scattered electron images show a contrast on the averaged atomic number, where the compositional heterogeneity is heavily emphasized. Upon cooling the ingot after its solidification, the temperature passes through the phase boundary between the body-centered cubic $\operatorname{Im} 3 m(\beta)$ phase and 
the hexagonal close-packed $P 6_{3} / m m c(\alpha)$ phase, causing the recrystallization of $\alpha$-platelets. This in turn creates the Widmanstätten patterns, which are similar to that observed in some furnace-cooled $\mathrm{Ti}-\mathrm{Zr}$ alloys with different compositions [9]. The typical lamellar width in the VAR-fabricated TiZr ingot was 5-50 $\mu \mathrm{m}$. TiZr alloys with such textures have been used as null-matrix alloys for neutron scattering.
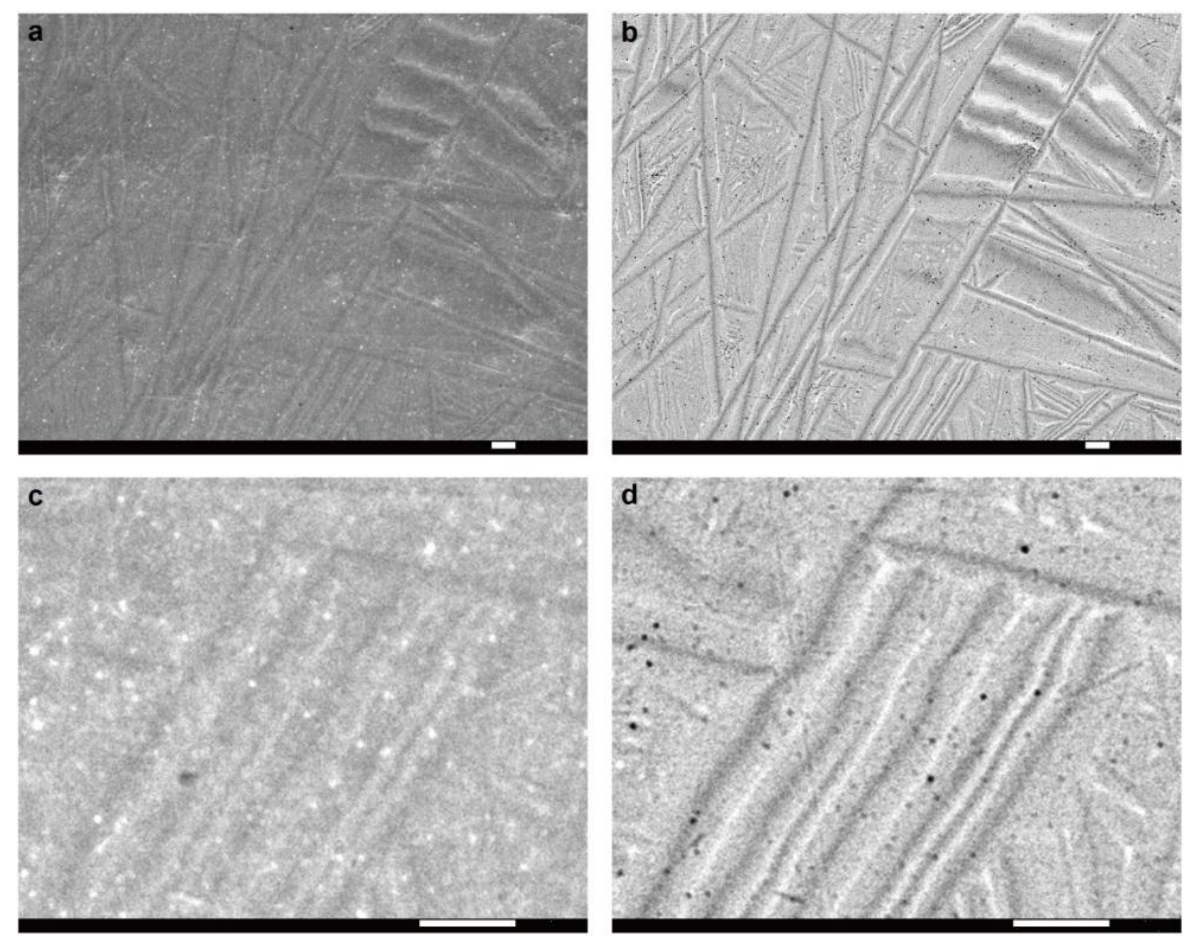

Figure 3. Cross-sectional (a) secondary-electron and (b) back-scattered-electron SEM images of the VAR-fabricated TiZr ingot before it was subjected to the mechanical forging. (c,d) Magnified images of areas in (a) and (b), respectively (magnification $=4 \times$ ). All scale bars indicate a length of $10 \mu \mathrm{m}$.

Figure 4 shows SEM images and elemental concentration maps of a polished cross section of the f-TiZr alloy, which was cut from a forged cylinder that was $20 \mathrm{~mm}$ in diameter. The very fine metallographic textures shown in these images should be effective to increase the hardness and strength of the metal [14], which has been repeatedly confirmed by previous studies, including those of Ti-based alloy systems [15-18]. This is also shown in our measurements of micro-Vickers hardness of the f-TiZr alloy at $H_{\mathrm{v}}=339$, which is significantly higher than that of non-forged $\mathrm{Ti}_{60} \mathrm{Zr}_{40}$ or $\mathrm{Ti}_{70} \mathrm{Zr}_{30}$ alloy with $H_{\mathrm{v}}=230 \sim 280$ [19]. The typical width of the $\alpha$-platelets in the f-TiZr alloy was $0.1-0.5 \mu \mathrm{m}$, which is approximately two orders of magnitude finer than that observed in the VAR-fabricated TiZr ingot (Figure 3 ). 

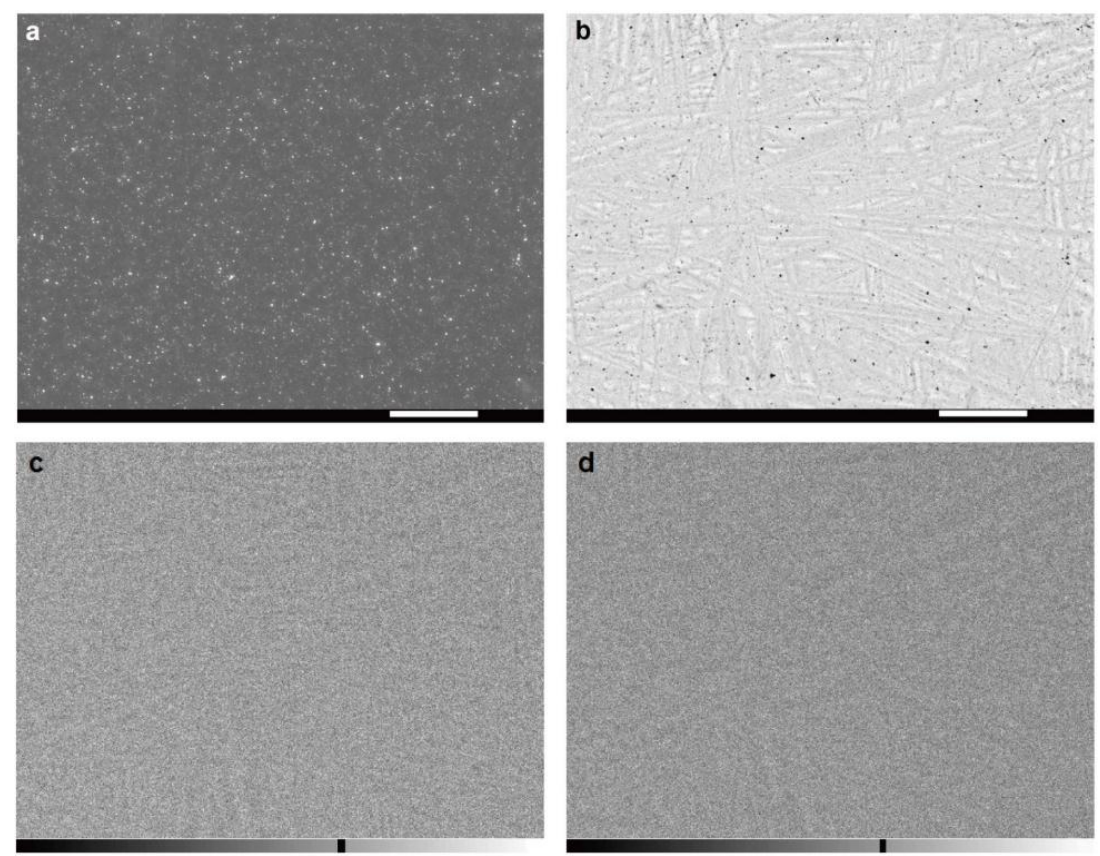

Figure 4. Cross-sectional (a) secondary-electron and (b) back-scattered-electron SEM images of the f-TiZr alloy along with the scale bars with $10 \mu \mathrm{m}$ in length. (c) $\mathrm{Ti}$ and (d) $\mathrm{Zr}$ concentration maps of the f-TiZr alloy obtained by EDS along with intensity scale bars (below each image where the left-end color shows no intensity) and averaged intensity (black line in each bar). All these images have identical spacial scale to that of Figure 3c,d.

Figure 5 shows a typical XRD pattern of the f-TiZr alloy. All of the reflections were successfully indexed to those of the $\alpha$-phase, as indicated by the indices of each peak. A significant broadening of these reflections is consistent with a large internal strain within the $\alpha$-platelets; this strain is essential for extensively hardening $\alpha$-Ti alloys $[9,19,20]$. Figure 6 shows the NPD patterns of the f-TiZr alloy. The patterns show that there are no diffraction peaks from the f-TiZr for the range of $d$-values measured. Therefore, we have confirmed that the forging process performed on the TiZr alloy has not degraded its null-scattering properties.

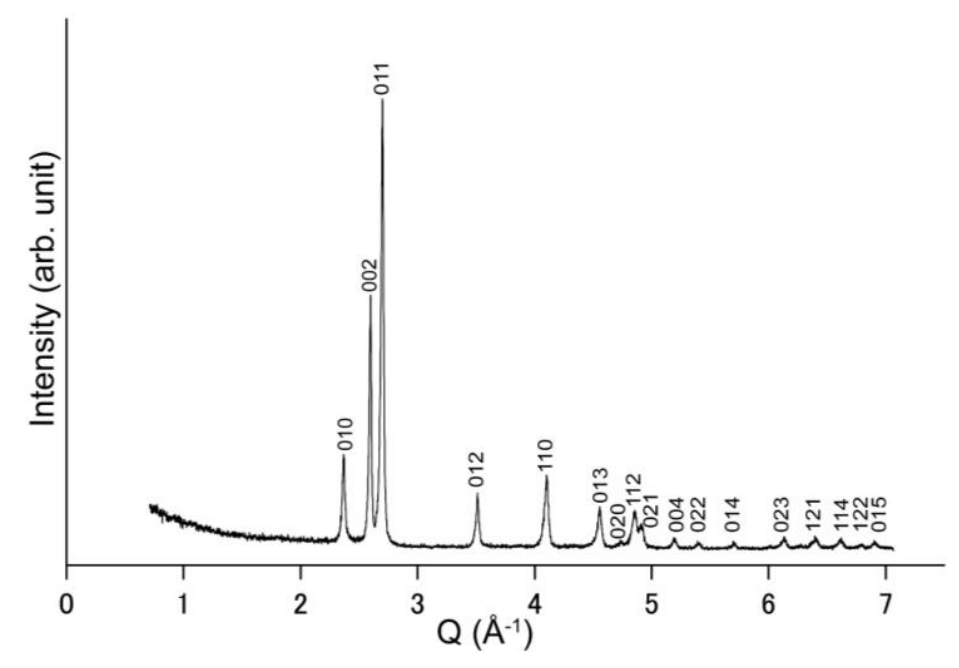

Figure 5. X-ray diffraction (XRD) pattern of the f-TiZr alloy. The Miller indices of the $\alpha$-phase are shown next to the corresponding reflections. 

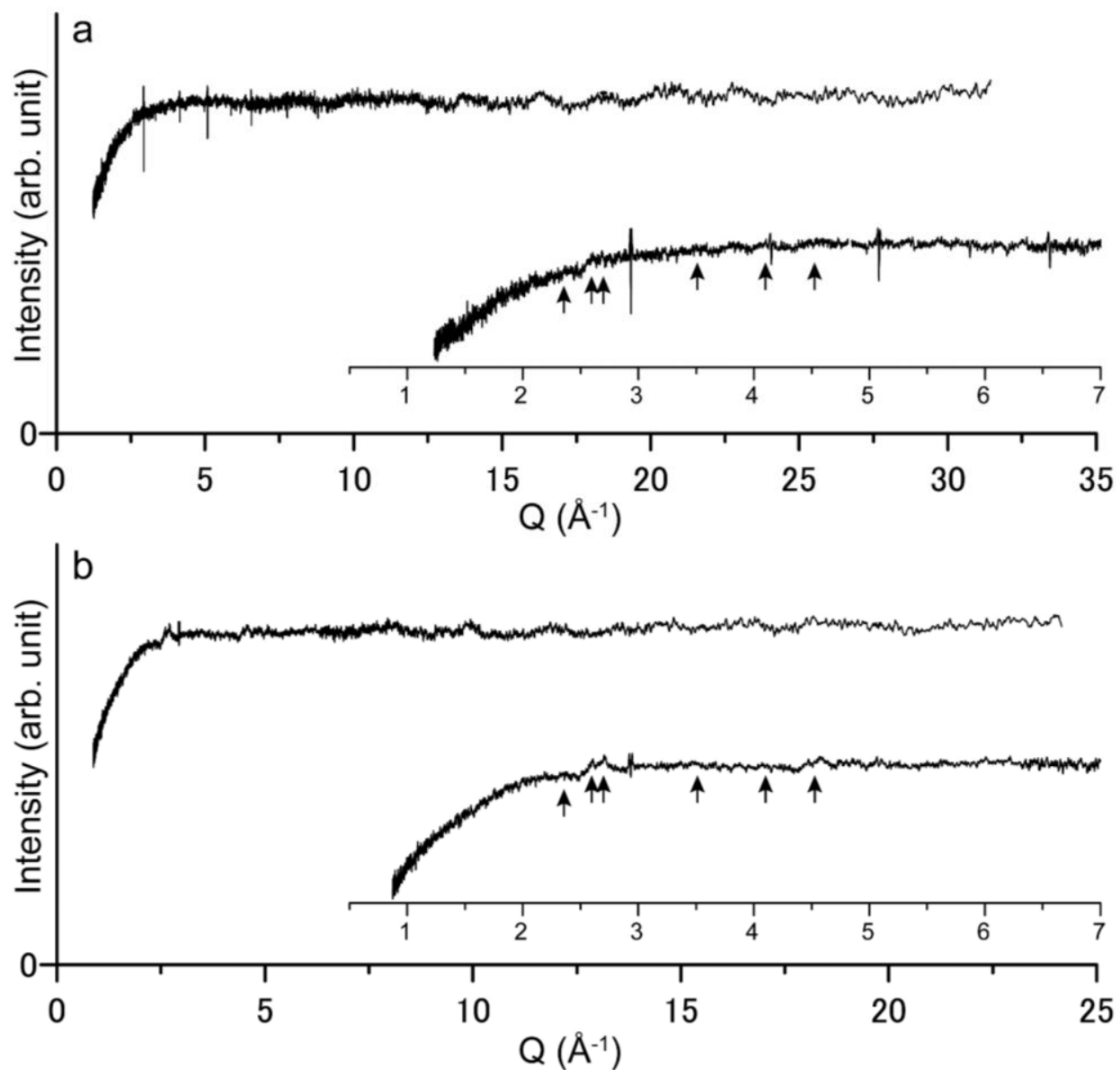

Figure 6. Neutron powder diffraction (NPD) patterns of the f-TiZr alloy: (a) NPD pattern from the high-resolution bank $(\Delta d / d \approx 0.16 \%)$ and $(\mathbf{b})$ that from the special environment bank $(\Delta d / d \approx 0.5 \%)$. The intensities of both profiles initially increase with increasing $Q$, which is due to inelastic scattering. The inset profiles show the lowest $Q$ regime which have the same vertical scale with the main figures. Arrows under both of the inset profiles indicate the positions of representative Bragg reflections that are observable when TiZr has a non-ideal composition; their indices include 010, 002, 011, 012, 110, and 013 for the $\alpha$ phase (see Figure 5). These Bragg reflections could be more evident for the TiZr used in the previous research (i.e., Figure 2.5 in Reference [1]). A few spurious, narrow peaks involving both negative and positive anomalies present in the profiles (at 2.93, 4.16 and $5.11 \AA^{-1}$ ), which were not completely removed by the background-subtraction procedures. These are caused by the diffraction of the sample holder, which was composed of $V$.

Figure 7 shows SEM images and elemental concentration maps of the annealed f-TiZr alloy. The width of the lamellae is smaller than $10 \mu \mathrm{m}$, and thus, the lamellae have not returned to the original dimensions of the VAR-fabricated TiZr ingot. On the other hand, its well-ordered metallographic texture is similar to that of the VAR-fabricated TiZr ingot or a furnace-cooled alloy [9]. The measured micro-Vickers hardness of the annealed f-TiZr was $H_{\mathrm{v}}=279$, which was reduced from the original hardness of the f-TiZr $\left(H_{\mathrm{v}}=339\right)$. 

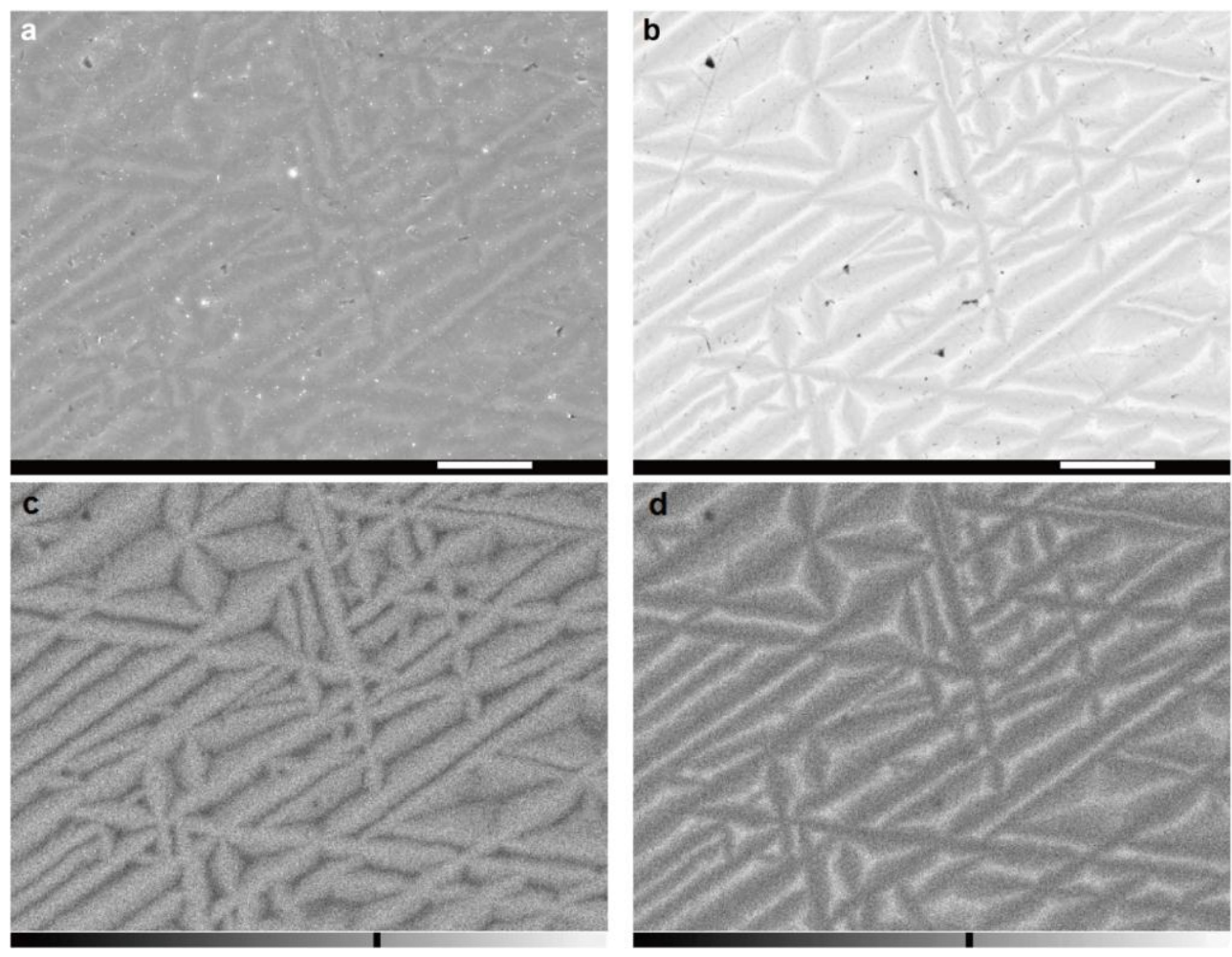

Figure 7. Cross-sectional (a) secondary-electron and (b) back-scattered-electron SEM images of the annealed f-TiZr alloy along with the scale bars with $10 \mu \mathrm{m}$ in length. (c) Ti and (d) $\mathrm{Zr}$ concentration maps of the annealed f-TiZr alloy obtained by EDS along with intensity scale bars (below each image where the left-end color shows no intensity) and averaged intensity (black line in each bar). All these images have identical spacial scale to that of Figure 3c,d.

\section{Application of the f-TiZr Alloy}

A series of experiments were conducted by fabricating deformable metallic gaskets from the f-TiZr alloy, and static pressures greater than $15 \mathrm{GPa}$ were successfully generated without the gaskets fracturing. We used a newly designed high-pressure cell that was optimized for neutron-scattering measurements with strong pulsed-neutron sources [21,22]. In brief, two anvils composed of single-crystal moissanite and sintered polycrystalline diamond with $5 \mathrm{~mm}$ culets were used to compress a powdered $\mathrm{Mg}(\mathrm{OD})_{2}$ sample, which was confined within the f-TiZr gasket (initial thickness $=1 \mathrm{~mm}$ ) and then compressed between the two anvils to $9 \mathrm{GPa}$. The total sample volume compressed to this high pressure was approximately $3 \mathrm{~mm}^{3}$, which was sufficient for performing a precise structure refinement via NPD at J-PARC. The gasket was recovered after this compression experiment, and it is shown in Figure 8. Similar to the other successful cases, the gasket has not cracked, even after the extensive deformation.

The annealed f-TiZr alloy was also applied to high-pressure NPD measurements with various types of anvil geometry, the results of which were partially published elsewhere [23]. 


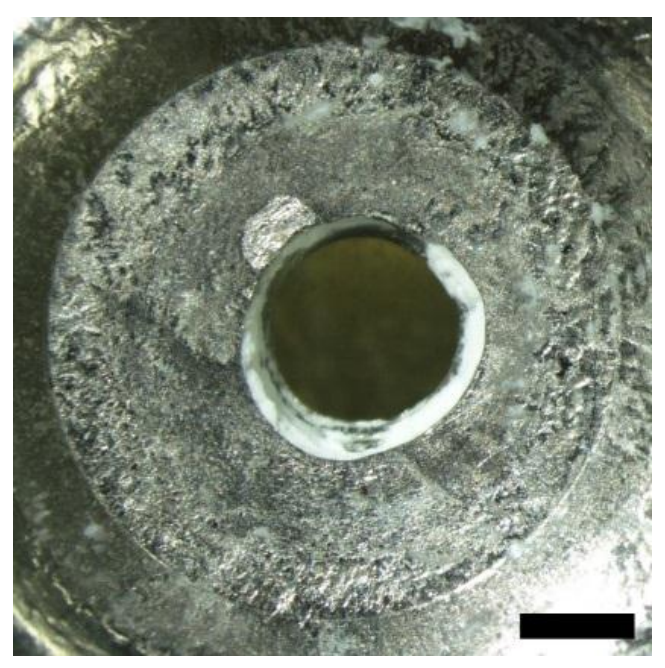

Figure 8. The recovered f-TiZr gasket, which experienced extensive deformation in the high-pressure cell. It was used to compress a powdered $\operatorname{Mg}(\mathrm{OD})_{2}$ sample to $9 \mathrm{GPa}$. The scale bar indicates a length of $1 \mathrm{~mm}$.

\section{Conclusions}

By applying extensive mechanical deformation at high temperatures to a TiZr alloy, the metallographic textures of the alloy became much finer, with the lamellae width decreasing by two orders of magnitude. The mechanical hardness of the TiZr was increased because of this change in the metallographic texture, which suggests that its strength was also increased, as previously observed in the general Ti-Zr two-component alloy system [9]. This f-TiZr alloy was successfully applied to experiments involving the generation of high static pressures, which requires the extensive deformation of the hard metal. In addition, the f-TiZr alloy was also used to fabricate a thin-walled micro-sample container, which enabled us to obtain a background-free NPD pattern with a pulsed-neutron source [24,25]. The successful application of the f-TiZr alloy has demonstrated that forged TiZr products may play a complementary role in future neutron scattering research.

\section{Acknowledgments}

The authors wish to thank T. Iikubo at Daido Bunseki Research Co, Ltd. and T. Matsumoto at the University of Tokyo for providing technical assistance. The machine time for the pulsed-neutron diffraction experiments was provided through the J-PARC MLF user program (No. 2013B0279). $\mathrm{N}$. Tomioka at Okayama University is acknowledged for helping these experiments. Four anonymous referees are acknowledged for their comments to improve the manuscript. This work was supported in part by JSPS Grants-in-Aid for Scientific Research (No. 19GS0205, 26287135 and 15K13593).

\section{Author Contributions}

T.O. wrote this manuscript and performed the experiments to obtain all of the results except for the neutron diffraction patterns. T.O., A.H., and T.I. performed the experiments to obtain the neutron diffraction patterns. All authors contributed to the interpretation of the experimental data. 


\section{Conflicts of Interest}

The authors declare no conflicts of interest.

\section{References}

1. Klotz, S. Techniques in High Pressure Neutron Scattering; CRC Press: Boca Raton, FL, USA, 2012.

2. Smith, J.H.; Vance, E.R.; Wheeler, D.A. A null-matrix alloy for neutron diffraction. J. Phys. E Sci. Instrum. 1968, 1, 945-947.

3. Sidhu, S.S.; Heaton, L.; Zauberis, D.D.; Campos, F.P. Neutron diffraction study of titanium-zirconium system. J. Appl. Phys. 1956, 27, 1040-1042.

4. Besson, J.M.; Nelmes, R.J.; Hamel, G.; Loveday, J.S.; Weill, G.; Hull, S. Neutron powder diffraction above 10 GPa. Phys. B Condens. Matter 1992, 180-181, 907-910.

5. Klotz, S.; Besson, J.M.; Hamel, G.; Nelmes, R.J.; Loveday, J.S.; Marshall, W.G.; Wilson, R.M. Neutron powder diffraction at pressures beyond 25 GPa. Appl. Phys. Lett. 1995, 66, 1735-1737.

6. Xu, J.; Ding, Y.; Jacobsen, S.D.; Mao, H.K.; Hemley, R.J.; Zhang, J.; Qian, J.; Pantea, C.; Vogel, S.C.; Williams, D.J.; et al. Powder neutron diffraction of wustite $\left(\mathrm{Fe}_{0.93 \mathrm{O}}\right)$ to $12 \mathrm{GPa}$ using large moissanite anvils. High Press. Res. 2004, 24, 247-253.

7. Williams, D.N.; Wood, R.A.; Jaffee, R.I.; Ogden, H.R. The effects of zirconium in titanium-base alloys. J. Less Common Met. 1964, 6, 219-225.

8. Zeidler, A.; Guthrie, M.; Salmon, P.S. Pressure-dependent structure of the null-scattering alloy Ti ${ }_{0.676} \mathrm{Zr}_{0.324 .}$. High Press. Res. 2005, 35, 239-246.

9. Imgram, A.G.; Williams, D.N.; Ogden, H.R. Tensile properties of binary titanium-zirconium and titanium-hafnium alloys. J. Less Common Met. 1962, 4, 217-225.

10. Meyers, M.A.; Mishra, A.; Benson, D.J. Mechanical properties of nanocrystalline materials. Prog. Mater. Sci. 2005, 51, 427-556.

11. Sakai, T.; Belyakov, A.; Kaibyshev, R.; Miura, H.; Jonas, J.J. Dynamic and post-dynamic recrystallization under hot, cold and severe plastic deformation conditions. Prog. Mater. Sci. 2014, 60, 130-207.

12. Blacktop, J.; Crangle, J.; Argent, B.B. The $\alpha \rightarrow \beta$ transformation in the Ti-Zr system and the influence of additions of up to 50 at. \% Hf. J. Less Common Met. 1985, 109, 375-380.

13. Ishigaki, T.; Hoshikawa, A.; Yonemura, M.; Morishima, T.; Kamiyama, T.; Oishi, R.; Aizawa, K.; Sakuma, T.; Tomota, Y.; Arai, M.; et al. IBARAKI materials design diffractometer (iMATERIA)-Versatile neutron diffractometer at J-PARC. Nucl. Instrum. Meth. A 2009, 600, 189-191.

14. Hall, E.O. Yield Point Phenomena in Metals and Alloys; Plenum Press: New York, NY, USA, $1970 ;$ p. 316.

15. Herebtsov, S.; Lojkowski, W.; Mazurb, A.; Salishcheva, G. Structure and properties of hydrostatically extruded commercially pure titanium. Mater. Sci. Eng. A 2010, 527, 5596-5603. 
16. Salishchev, G.A.; Galeev, R.M.; Malysheva, S.P.; Zherebtsov, S.V.; Mironov, S.Y.; Valiakhmetov, O.R.; Ivanisenko, E.I. Formation of submicrocrystalline structure in titanium and titanium alloys and their mechanical properties. Met. Sci. Heat Treat. 2006, 48, 63-69.

17. Valiev, R.Z.; Alexandrov, I.V. Paradox of strength and ductility in metals processed by severe plastic deformation. J. Mater. Res. 2002, 17, 5-8.

18. Kobayashi, Y.; Tanaka, Y.; Matsuoka, K.; Kinoshita, K.; Miyamoto, Y.; Murata, H. Effect of forging ratio and grain size on tensile and fatigue strength of pure titanium forgings. J. Soc. Mater. Sci. Jpn. 2005, 54, 66-72.

19. Kobayashi, E.; Matsumoto, S.; Doi, H.; Yoneyama, T.; Hamanaka, H. Mechanical properties of the binary titanium-zirconium alloys and their potential for biomedical materials. J. Biomed. Mater. Res. 1995, 29, 943-950.

20. Dobromyslov, A.V.; Taluts, N.I. Structure investigation of quenched and tempered alloys of the Zr-Ti system. Phys. Met. Metallogr. 1987, 63, 114-120.

21. Okuchi, T.; Sasaki, S.; Ohno, Y.; Abe, J.; Arima, H.; Osakabe, T.; Hattori, T.; Sano-Furukawa, A.; Komatsu, K.; Kagi, H.; et al. Neutron powder diffraction of small-volume samples at high pressure using compact opposed-anvil cells and focused beam. J. Phys. Conf. Ser. 2012, doi:10.1088/1742-6596/377/1/012013.

22. Okuchi, T.; Yoshida, M.; Ohno, Y.; Tomioka, N.; Purevjav, N.; Osakabe, T.; Harjo, S.; Abe, J.; Aizawa, K.; Sasaki, S. Pulsed neutron powder diffraction at high pressure by a capacity-increased sapphire anvil cell. High Press. Res. 2013, 33, 777-786.

23. Okuchi, T.; Tomioka, N.; Purevjav, N.; Abe, J.; Harujo, S.; Gong, W. Structure refinement of sub-cubic-mm volume sample at high pressures by pulsed neutron powder diffraction: Application to brucite in an opposed anvil cell. High Press. Res. 2014, 34, 273-280.

24. Purevjav, N.; Okuchi, T.; Tomioka, N.; Abe, J.; Harjo, S. Hydrogen site analysis of hydrous ringwoodite in mantle transition zone by pulsed neutron diffraction. Geophys. Res. Lett. 2014, 41, 6718-6724.

25. Tomioka, N.; Okuchi, T.; Purevjav, N.; Abe, J.; Harjo, S. Hydrogen sites in the dense hydrous magnesian silicate phase E: A pulsed-neutron powder diffraction study. Phys. Chem. Miner., in press.

(C) 2015 by the authors; licensee MDPI, Basel, Switzerland. This article is an open access article distributed under the terms and conditions of the Creative Commons Attribution license (http://creativecommons.org/licenses/by/4.0/). 